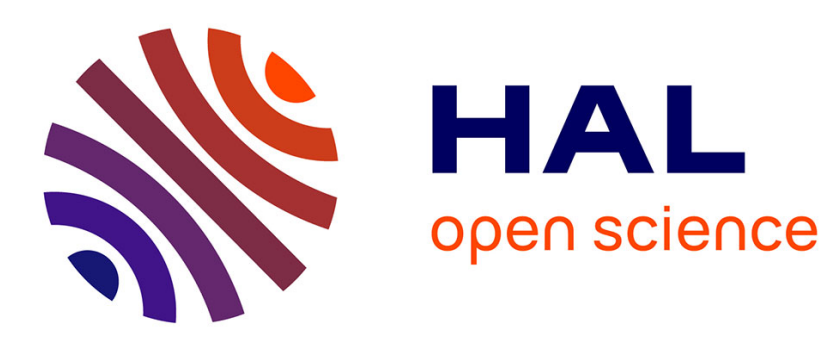

\title{
Combined effect of YORP and collisions on the rotation rate of small Main Belt asteroids
}

\author{
F. Marzari, A. Rossi, D.J. Scheeres
}

\section{To cite this version:}

F. Marzari, A. Rossi, D.J. Scheeres. Combined effect of YORP and collisions on the rotation rate of small Main Belt asteroids. Icarus, 2011, 214 (2), pp.622. 10.1016/j.icarus.2011.05.033 . hal-00786881

\section{HAL Id: hal-00786881 \\ https://hal.science/hal-00786881}

Submitted on 11 Feb 2013

HAL is a multi-disciplinary open access archive for the deposit and dissemination of scientific research documents, whether they are published or not. The documents may come from teaching and research institutions in France or abroad, or from public or private research centers.
L'archive ouverte pluridisciplinaire HAL, est destinée au dépôt et à la diffusion de documents scientifiques de niveau recherche, publiés ou non, émanant des établissements d'enseignement et de recherche français ou étrangers, des laboratoires publics ou privés. 


\section{Accepted Manuscript}

Combined effect of YORP and collisions on the rotation rate of small Main Belt asteroids

F. Marzari, A. Rossi, D.J. Scheeres

PII:

S0019-1035(11)00206-5

DOI:

10.1016/j.icarus.2011.05.033

Reference:

YICAR 9840

To appear in:

Icarus

Received Date: $\quad 3$ February 2011

Revised Date: $\quad 26$ May 2011

Accepted Date: $\quad 26$ May 2011

Please cite this article as: Marzari, F., Rossi, A., Scheeres, D.J., Combined effect of YORP and collisions on the rotation rate of small Main Belt asteroids, Icarus (2011), doi: 10.1016/j.icarus.2011.05.033

This is a PDF file of an unedited manuscript that has been accepted for publication. As a service to our customers we are providing this early version of the manuscript. The manuscript will undergo copyediting, typesetting, and review of the resulting proof before it is published in its final form. Please note that during the production process errors may be discovered which could affect the content, and all legal disclaimers that apply to the journal pertain. 


\title{
Combined effect of YORP and collisions on the rotation rate of small Main Belt asteroids.
}

\author{
F. Marzari
}

Dipartimento di Fisica, Università di Padova, 35131 Padova, Italy

E-mail: francesco.marzari@pd.infn.it

A. Rossi

IFAC-CNR \& ISTI-CNR, 50019 Sesto Fiorentino (FI), Italy

E-mail: Alessandro.Rossi@isti.cnr.it

\section{D.J. Scheeres}

Department of Aerospace Engineering Sciences,

The University of Colorado, Boulder, Colorado 80309-0429 USA

E-mail: scheeres@colorado.edu

June 3, 2011 
Please, send editorial communications and proofs to:

Francesco Marzari

Dipartimento di Fisica

Via Marzolo, 8

35131 padova, Italy

Tel: $39-049-8277190$

Fax: 39-049-8277102

E-mail: marzari@pd.infn.it

Manuscript pages: 31

Figures: 9

Running title: YORP and collisions

Keywords: ASTEROIDS, ROTATION; ROTATIONAL DYNAMICS; COLLISIONS. 


\begin{abstract}
The rotation rate distribution of small Main Belt asteroids is dominated by YORP and collisions. These mechanism act differently depending on the size of the bodies and give rise to non-linear effects when they both operate. Using a Monte Carlo method we model the formation of a steady state population of small asteroids under the influence of both mechanisms and the rotation rate distribution is compared to the observed one as derived from Pravec et al. (2008). A better match to observations is obtained with respect to the case in which only YORP is considered. In particular, an excess of slow rotators is produced in the model with both collisions and YORP because bodies driven to slow rotation by YORP have a random walk-like evolution of the spin induced by repeated collisions with small projectiles. This is a dynamical evolution different from tumbling and it lasts until a large impact takes the body to a faster rotation rate. According to our model, the rotational fission of small asteroids is a very frequent event and might explain objects like P/2010 A2 and its associated tail of millimeter-sized dust particles. The mass loss during fission of small asteroids might significantly influence the overall collisional evolution of the belt. Fission can in fact be considered as an additional erosion mechanism, besides cratering and fragmentation, acting only at small diameters.
\end{abstract}




\section{Introduction}

The rotation rates of small asteroids in the Main belt, mostly deduced from periodic variations in their brightnesses, are controlled by mutual collisions and YORP. Any model for the interpretation of their mean rotation rate and dispersion, as derived from lightcurve compilations, must account for both these mechanisms. During a collision between a small projectile and the target asteroid, angular momentum is transferred from the orbital motion of the projectile to the spin of the target. In addition, if some fragments are able to escape the gravity of the target, some mass and angular momentum may be lost. The amount of change in the rotation rate and spin direction depends not only on the energy of the impact (size and velocity of the projectile) but also on the geometry of the encounter. Off-axis cratering events are very effective in changing the spin state of an asteroid. These impulsive variations in the rotation rate, which constantly occurs in the Main Belt because of the dense population of bodies, may significantly interfere with YORP (Rubincam 2000, Vokrouhlický and Čapek 2002, Čapek and Vokrouhlický 2004) by changing the rotation parameters, the phase of the YORP cycle, and the torque itself. In addition, energetic cratering events alter the superficial features of the asteroid causing a significant change in the recoil force as the absorbed heat is re-emitted. According to Statler 
(2009), alterations to the small-scale topography may even reverse the sign of the YORP torque.

During the lifetime of an asteroid, in between two breakup events that would significantly reduce its shape and reset its rotation state, the variation of the spin rate will be determined by the interplay between YORP and cratering impacts. The combined effect of the two mechanisms is not necessarily a linear combination of the independent contributions from either YORP or collisions. In fact, collisions affect the YORP evolution by changing its phase and parameters while YORP changes the rotation rate in between two subsequent collisions leading to a different rotational configuration at the next collision. As a consequence, we expect a complex behavior of the spin under the action of the two mechanisms and we find that this is particularly relevant for slow rotators. In fact, for slowly spinning bodies repeated collisions can temporarily halt the YORP evolution triggering a period of slow rotation. On the other hand, when YORP is gently driving the body towards the breakup limit a collision may abruptly send the body beyond its spin limit causing immediate fission. To model the combined effects of YORP and collisions, we have developed a computer model which simulates both the YORP and collisional evolution of the rotation rate for asteroids of any size. Starting with a population of asteroids with a fixed 
size distribution and a given initial rotation rate distribution, we can derive the relaxed state of the spins and infer a distribution that can be compared to observations. In this way we can better constrain the parameters that determine the spin evolution and, possibly, the initial distribution as outcome of collisions for small asteroids. In addition, keeping track of the rotation rate of the individual bodies, we can estimate the number of objects spun beyond the fission limit by the combined YORP and collision effects. The frequency with which bodies of a given diameter are accelerated beyond the breakup limit can be estimated and tested against the occurrence of events like the formation of a cometary tail for body P/2010 A2. Fission appears a likely mechanism for such phenomena.

In Section 2 we describe the numerical model we have developed which includes both YORP and cratering events. In Section 3 we illustrate the evolution of individual bodies as a function of time. In Section 4 we apply a Monte Carlo method to model a full population of asteroids and in Section 5 we describe the results of our simulations and their dependence on the model parameters. Section 6 is devoted to the discussion of rotational fission as a source of objects like $\mathrm{P} / 2010$ A2 while in Section 7 we discuss the results and future perspectives. 


\section{The numerical model}

In the next section we describe the details of the Monte Carlo numerical model used to simulate the evolution of the spin rate of a population of asteroids in the main belt under the coupled action of YORP and collisions.

\subsection{Algorithm modeling the collisional change of the asteroid spin}

To model the evolution of the spin of a single asteroid due to repeated collisions with other asteroids of the Main Belt we first define its initial radius $r$, mass $M$ and angular momentum vector $L$. The population of potential impactors is then derived from the SDSS (Sloan Digital Sky Survey) size distribution of asteroids (Ivezic et al. 2001) and is distributed over logarithmic size bins. Using Poisson statistics, we compute both the number of collisions that the asteroid has with projectiles coming from each size bin and their timing, using the intrinsic probability of collision for the Main Belt $<P_{i}>$ (Farinella and Davis 1992, Bottke et al. 1994, Vedder 1996, 1998). At this stage of the model we have a list of random impacts sorted in time which are characterized by the collisional time, projectile size and by an impact velocity which is randomly drawn from the distribution given in Bottke et al. (1994). The subsequent step consists in computing the change in $L$ due to all collisions. For each impact on the list we define an approach geometry 
which is randomly defined within the limits given by the orbital element distribution of asteroids in the Belt. The strongest constraint is on the velocity along the z-axis which cannot exceed that predicted by the average inclination of the Belt. After each impact, we add the angular momentum vector $L_{i}$ of the projectile to that of the target $L$. In this way we update the rotation rate and direction of the spin axis. If the impactor is too big for a cratering event, we assume that the target is shattered, we record the breakup and draw a new object from the initial distribution. We neglect the angular momentum taken away by fragments that may escape after the cratering. This approximation is good for the frequent low energy impacts, but is less precise for the few very energetic impact events. However, as stated above, these events are not really important for the overall evolution of the spin rate of small asteroids. The outcome of repeated collisions on the rotation rate is a random walk that tends to spread the initial spin distribution. The overall effects of shattering events on the spin distribution is not included in our model because anytime a body is fragmented, it is removed and substituted by a new one preserving no memory of the previous spin value. This allows us to keep our model population stationary. This is also a good approximation because for small asteroids the leading mechanism determing the spin evolution is YORP and, after a shattering event, any 
record of the pre-fragmentation YORP cycle is lost and the body is set in a new YORP state.

\subsection{YORP evolution}

In between two collisions the spin rate of an asteroid evolves under YORP. We follow the approach outlined in Scheeres (2007) and also adopted in Rossi et al. (2009). A non-dimensional YORP coefficient $C_{Y}$ is defined for the asteroid whose value, according to Scheeres (2007), is encompassed between -0.025 and 0.025 (see table 4 ). This coefficient depends on both the asteroid's shape and moment of inertia. It is noteworthy that the estimated values of $C_{Y}$ for the asteroids Apollo and YORP are 0.022 and 0.005 , respectively, based on values taken from (Kaasalainen et al. 2007 and Taylor et al. 2007). An initial value for $C_{Y}$ is drawn randomly for an asteroid at the beginning of the simulation. The rotational acceleration for the body in between two collisions is then computed as:

$$
\dot{\omega}=\frac{B G_{1} r}{A^{2} \sqrt{1-e^{2}} M} C_{Y}
$$

where $B$ is a Lambertian scattering coefficient, usually taken to equal $2 / 3$, $G_{1} \sim 1 \times 10^{14} \mathrm{~kg} \mathrm{~km} / \mathrm{s}^{2}$ is the solar radiation constant. The asteroid orbital parameters like semi-major axis $A$ (in $\mathrm{km}$ ) and eccentricity $e$ are initial parameters of the model. 
After any collision we update the obliquity of the body $\varepsilon$ according to the angular momentum variation. The YORP coefficient is also re-computed, to account for the change in obliquity, following the equation:

$$
C_{Y}^{\prime}=C_{Y}\left(3 \cos (\varepsilon)^{2}-1\right) / 2
$$

from Nesvorný and Vokrouhlický (2008). In our approximation we ignore the obliquity dynamics due to YORP and we consider only the impulsive changes due to collisions. We plan to include obliquity variations once we have a better developed analytical model for the complex rotational evolution of these bodies under YORP. A first approach to this theory is found in Cicalo and Scheeres (2010), however this does not address or predict overall spinup/spin-down of the body, which should also be an issue. The inclusion of YORP obliquity dynamics also implies that we must also model the thermal inertia of these bodies, as previous analytical predictions and numerical results have shown that the obliquity dynamics are quite sensitive to this parameter. As this will add additional uncertain parameters into our model, and should ideally only provide a randomization of the obliquities (which the impacts already essentially does), we feel that this addition is not needed for the current generation of our model.

If a collision is energetic, the changes in rotation rate and shape can alter the subsequent YORP cycle. For this reason, whenever a collision is violent 
enough to change the obliquity by a fraction $\delta \geq 0.2$ we draw for the body a new YORP coefficient $C_{Y}$ that will be used from then on. The change in $C_{Y}$ is ascribed to the modifications of the surface properties of the asteroid caused by the cratering (the formation of a new crater, moving boulders, shifting the barycenter etc., see Statler 2009).

If the asteroid approaches its critical rotation rate for breakup, we assume that its shape will be reconfigured because of boulders shifting, mass shedding and fission as discussed in Scheeres et al. (2007) and Jacobson and Scheeres (2011). The amount of body changes at breakup is a complex issue and the amount of mass erosion depends on the physical properties of the body itself. As a first rough approximation, we assume that the body survives and re-starts its YORP evolution with a constant torque that acts to spin the body up in the opposite direction (see Vokrouhlický et al. 2007).

In some cases, a collision can abruptly accelerate the spin of a body already driven close to the breakup limit by YORP driving it well well beyond the breakup limit. In this case, very different from a YORP-only more gentle evolution, we expect that the body is disrupted like in a fragmenting impact, implying that a more detailed modeling of this event is needed using numerical algorithms that model asteroids as an agglomerate of rigid particles (Richardson et al. 2000; Richardson et al. 2005; Sánchez and Scheeres 
2011). In our model, any time a collision spins up the body in a step-like way beyond the critical rate, we substitute it with a new one having a new random rotation rate. This is the same algorithm used to replace bodies which are fragmented by energetic projectiles.

\section{Evolution of single asteroids}

In Fig. 1 we illustrate the evolution of the rotation rate of individual asteroids having different sizes. As expected, for smaller asteroids the YORP cycles are faster but they can be interrupted and possibly reversed by collisions which, if sufficiently energetic, may alter the YORP parameters. Large steps are also observed due only to collisions as for some larger asteroids.

In our model we assume that the variation (and even reversal) of the YORP cycle depends on the amount of obliquity change. We arbitrarily set this limit to $\delta=0.2$. However, a collisional reshaping sufficient for altering the YORP coefficient $C_{Y}$ might occur also for less energetic impacts. As shown in Statler (2009), YORP is very sensitive to topographic features like moderate sized craters or boulders. A collision does create a new crater and if it is energetic enough the crater size may be comparable with the

body radius and significantly change the YORP evolution. In addition to that, the center of mass may be displaced and boulders can be produced 
in the impact. As a consequence, the assumption that collisions can alter the YORP evolution is well based. It is however not well defined how the coefficient $C_{Y}$ depends on the impact energy of cratering events. There is also an inherent stocasticity due to the crater formation process and the ejecta evolution.

\section{The Monte Carlo model and the evolution of the asteroid population}

Our model for the evolution of a single asteroid can be extended to a whole population of asteroids. A Monte Carlo approach is used with a structure similar to that described in Rossi et al. (2008) and Scheeres et al. (2004). A population of $10^{5}$ asteroids is initially generated. Their initial size distribution, ranging from $100 \mathrm{~m}$ to $20 \mathrm{~km}$, follows that given in O'Brien and Greenberg (2003) for the entire belt. The spin distribution of these putative asteroids is in agreement with the Maxwellian distributions presented in Fulchignoni et al. (1995) and Donnison and Wiper (1999). To each body of our sample we assign a value of semimajor axis and eccentricity which are used in the computation of the YORP torque. The orbital elements are selected randomly following the known Main belt orbital distribution. This population is evolved under the action of YORP and collisions. During the interval of time covered by the numerical model, an asteroid in our sample 
may undergo a collisional event able to disrupt the body. The size of the projectile needed to disrupt and disperse half of the parent body (which is the definition of a fragmentation event) is computed following Bottke et al. (2005). Whenever such an event occurs, a new body is generated with the Monte Carlo method so that the total number of bodies in our sample is constant. The driving reason for this choice is that the collisional evolution of larger asteroids is constantly refilling the population of smaller bodies. Cratering and fragmentation of large size asteroids create a bunch of small bodies that replace those disrupted in collisions and the population of small asteroids is then stationary. A value of $C_{Y}$ is also drawn randomly for the new body following a Gaussian distribution centered in 0.

As a caveat, we point out that our model does not model the full collisional evolution of asteroids and is then limited compared to other models like that described in Farinella et al. (1992). However, the dominant mechanism for the spin evolution of small asteroids is YORP, while collisions can be considered as a perturbation of the YORP induced cycles. This perturbation is significant since it frequently changes the YORP coefficient by altering the superficial features of the body, possibly reversing the YORP evolution. At the same time, in the lower limit of the rotation state collisions interact non-linearly with YORP causing significant effects. In this 
light, our collisional model can be managed with some approximations like neglecting the escaping fragments and not fully exploring the parameters of the collisional physics. In their paper, Farinella et al. 1992 explored in detail both the collisional and rotational evolution of asteroids but, since at that time YORP was not considered a relevant effect, they did not include it as a source of spin change. As a consequence, they see in their outcome a dependence on the collisional parameters. In our case, we use a simplified approach and we neglect fragmentation since any time a body is fragmented, it is assumed to move to a different size bin and be replaced by a new body. In this way we keep the population fixed to the SSDS slope.

An additional simplification of our model is in not accounting for the possible tumbling state that an asteroid might enter when its rotation rate is slow either because of YORP (Vokrouhlický et al. 2007) or caused by gentle collisions. In the former case Vokrouhlický et al. 2007 have shown that at the YORP reversal the body accelerates but preserves a tumbling state. However, the internal stresses due to wobbling cause dissipation of energy which will eventually drive the rotation back towards a stable rotation mode. The persistence of the tumbling state depends on energy dissipation rates that are difficult to determine and depend on the shape, physical state and composition of the body (see for example Efroimsky and Lazarian, 2000). 
Our models where YORP is the only source of spin variation may not correctly describe the end state of rotation since we assume that the subsequent spin acceleration instantly occurs. However, even in this case the relevant parameter is the energy dissipation timescale.

The scenario when collisions are included is different. We will discuss this in more detail in the following section.

\section{Results}

With our model we evolve the population of small Main Belt asteroids over the age of the solar system. We first explore the effects of YORP and collisions separately and, finally, we run simulations where both effects are included. In Fig. 2 the cumulative distribution of the rotation rates is shown for the three different situations. Both YORP and collisions contribute independently to create a population of fast rotators while YORP is much more effective in slowing down the rotation rate. In the simulation where only YORP is included, a significant fraction of asteroids become slow rotators. When we include in the algorithm both YORP and collisions, the number of slow rotators is consistently increased. To compare our model results with observations we have to artificially bias our population in order to simulate the observational incompletness. We have followed the same method de- 
scribed in Rossi et al. (2009) and we have divided the diameter range in size bins and in each bin we have selected randomly from our model population a number of objects equal to that of the observed population. In Fig. 3 we compare our biased population with the observed cumulative distribution by Pravec et al. (2008). The numerical model with only the YORP effect included significantly underestimates the number of slow rotators observed in the real population. Even fast rotators are not well reproduced in this scenario. When the collisions are included in the model, the observational data are better matched and an excess of slow rotators is found. Since neither YORP or collisions alone are independently able to produce this large tail of slow rotators, it is an effect related to a non-linear coupling between the two mechanisms.

What happens is the following. Far from a slow rotation state, only the occasional energetic collisions are able to significantly change the rotation rate with a sudden step, while the effects of small impactors are easily absorbed by the overall evolution even if they can change the YORP coefficient and the rotation direction. On the other hand, when the rotation rate of an object is smoothly slowed down by YORP reaching longer periods, the amount of angular momentum change imparted by collisions becomes comparable to the total angular momentum of the body. This keeps the asteroid 
"lingering" in a slow rotation state for a comparatively large time, leading to the observed excess. It is noteworthy that we are not modeling a tumbling state, rather the spin follows a sort of random walk driven by low energy impacts until an impact with a large projectile significantly accelerates the spin, stabilizing the object against less energetic impacts again. At this point, the spin rate can be smoothly accelerated by YORP and the asteroid enters the "normal" YORP cycle. This effect happens mainly for the larger objects in our sample (around $5 \mathrm{~km}$ of diameters) and is shown in Fig. 4. For comparison, in Fig. 5 we illustrate the evolution of the same objects under the influence of YORP only (i.e., in a simulation where collisions are neglected). In this latter case only the smooth YORP cycles are observed and no excess of slow rotators is observed in the cumulative distribution. For smaller objects the YORP cycles are so short and the perturbation induced by collision so frequent and comparatively large, that the body is rapidly jumping between the two extreme states of slow and fast rotation and no accumulation at slow rotation rates is observed. E.g., in Fig. 6, the fast evolution of the spin for a small (61 meter diameter) object, under the effect of YORP and collisions, is shown. The YORP evolution is so rapid that it prevents any lingering at slow spins.

This collisional lingering caused by frequent gentle collisions may inter- 
fere with the YORP induced tumbling state, either causing tumbling before YORP itself does (see Vokrouhlický et al. 2007), or by altering its evolution. The effects of collisions on the rotation rate of a tumbling asteroid is a complex subject and could be modeled by directly solving the equations of the orbital and rotational motion as in Vokrouhlický et al. (2007), introducing a step change anytime a collision occurs. It appears unlikely that tumbling might prevent collisions to extend the period of slow rotation by gentle kicks in different directions, but a detailed treatment of this effect is needed.

Another example of non-linear interaction is observed when an asteroid is accelerated by YORP close to the breakup limit. A collision occurring at this stage will either slow the body down with a step change and lead it to a different YORP evolution, or it will take the asteroid immediately beyond the breakup limit. As mentioned above, in this case the asteroid will be substituted by a new body with a new random rotation rate in our simulation. This combination of YORP and collisions reduce the number of asteroids with a spin rate close to the breakup limit compared to a scenario with only YORP. This phenomenon is observed in Fig. 3 where the number of fast rotators is lower when both collisions and YORP are included in the model compared to the case with only YORP. 


\subsection{Dependence on the size distribution at small diameters and on the parameter $\delta$.}

The size distribution is a poorly constrained parameter when modeling the evolution of small asteroids in the main belt. We have used in our modeling the SDSS size distribution but the recent SKADS survey by Gladman et al. (2009) seems to point to a somewhat shallower slope at small diameters. For this reason we have tested different slopes for the size distribution in our model with the results are shown in Fig. 7. When the slope is shallower, the projectile population is reduced and the lifetime of the asteroids we consider is longer. Consequently, more bodies experience the lingering at low rotation rates and the population of slow rotators is considerably increased. It is also true that the number of projectiles that can keep the bodies lingering at low rotation rates is decreased, but according to the numerical model this is not enough to halt the production of slow rotators.

One might argue that from our model we could derive constraints on the size distribution of main belt asteroids at the small size end by trying to match the rotation rates. However, we are still far from doing it. While the steeper slope of 2.1 gives a better match to observations on the slow rotators side, it underestimates the number of asteroids with intermediate spins in favor of slow and fast rotators. Additional aspects may be missing in our 
model, like a dependence of the asteroid shape on the size and possibly the consequences of the breakup processes that produces small bodies. This might introduce an additional size dependence on the YORP coefficient which could affect the non-linear interplay between collisions and YORP and change the steady state spin distribution.

An additional parameter of our model is $\delta$, the amount of change in obliquity that leads to a re-drawing of the YORP coefficient. Its value is not constrained by present theories of YORP apart from the fact that it must be small. We sampled 3 different values of this coefficient to test its influence on the overall distribution of the spin rates. In Fig. 8 we illustrate the changes in the cumulative spin distribution due to different values of $\delta$. The differences in the distributions for $\delta=0.1,0.2,0.3$ appear to be mostly due to random effects.

\section{Rotational fission as source of objects like $\mathrm{P} / 2010$ A2}

Observations by Jewitt et al. (2010) of the peculiar object P/2010 A2, a small body with a diameter of approximately $120 \mathrm{~m}$ orbiting in the inner asteroid belt, have revealed an extended tail of dust particles. Snodgrass et al. (2010) proposed that the tail is made up of debris from a cratering event traced back to early 2009. In addition to the collision hypothesis, 
Jewitt also suggested an alternative explanation assuming that the body was partially disrupted by rotational bursting. Our model predicts that spinning up by YORP beyond the limit of cohesion is indeed a more frequent event compared to an equivalent cratering. Material at the surface of the asteroids may reach ejection speeds separating from the body at the equator (Walsh et al. 2008) or may undergo fission followed by mutual interactions (Jacobson and Scheeres 2011) that could create a dusty tail similar to that detected by Jewitt et al. (2010). Mass shedding has been also proposed as an additional mechanism for slowing down rapidly spinning asteroid (Bottke et al., 2002; Scheeres et al., 2006; Walsh and Richardson, 2006b).

In Fig. 9 we show the number of expected fission events per year due to YORP for asteroids in the belt as a function of their diameter. It is noteworthy that the number of fissioning bodies is larger than the number of fragmentation events for bodies smaller than $2 \mathrm{~km}$. In particular, for a $100 \mathrm{~m}$ size body in the belt we find about 360 asteroids per year spinning beyond the critical rate and possibly causing mass shedding. This is a significant number and it may explain the observations of P/2010 A2 and even suggest that fission may be the preferred source of a dusty tail compared to cratering. According to Jacobson and Scheeres (2011), material entering orbit via rotational fission will always escape (unless the mass ratio between 
the components is greater than 0.2 ) with two effects: the formation of a dusty tail and the erosion of the original body. If a significant number of bodies are involved in such a fission process, their constant erosion might affect the size distribution of asteroids at small diameters. This would be an independent mechanism, apart from collisions, causing a reduction in the number of small asteroids. The -3.5 collisional equilibrium slope of the differential size distribution of asteroids inferred on a theoretical ground by Dohnanyi (1969) for a collisionally relaxed system would no longer be a good approximation as it neglects the contribution from asteroidal fission of small asteroids. Numerical collisional evolution models used to simulate the erosion of the belt in addition to cratering and fragmentation should also include fission to match observations. To estimate the influence of this mechanism we need a detailed model of what happens to an asteroid when it is accelerated beyond the critical rotation rate. In this way we can devise a general approach allowing us to draw a statistical outcome (fission, binary formation, amount of erosion etc..) for any given object accelerated by YORP beyond the critical value. This work is in progress and will allow one to derive predictions on the number of binaries and other morphology properties. A more complex approach is required if we want to estimate the effect of fissioning erosion on the size distribution of asteroids. 


\section{Discussion and Conclusions}

The rotational evolution of small asteroids in the Main Belt is dominated by YORP and collisions. Both these physical mechanisms can significantly alter the spin rate of a body and change its primordial value, either that achieved after the breakup event that created the body itself or that produced during the formation process for unshattered bodies. However, the two mechanisms are not acting independently since they may interfere with each other and give origin to non-linear effects. First of all collisions can modify the small scale topography of an asteroid and, according to Statler (2009), change the YORP cycle. In addition, close to the two limiting states of a rotating body the non-linearity is particularly strong. When a small asteroid is in a slow rotation state, small scale cratering events cause a random walk in the spin period which lasts until a large projectile hits the body restoring the YORP cycle. This causes an increase in the number of slow rotators in a steady state population. Moreover, when driven close to the breakup limit by YORP, an energetic collision may lead the body beyond the critical rotation rate causing its disruption. A reduction in the number of surviving fast rotators is then expected. However, the overall spin distribution at the fast rotation end is mostly due to YORP.

With our model based on a Monte Carlo approach, we account for both 
YORP and collisions and we show that their combined effects lead to a better match to the observed rotation rate distribution of small asteroids, compared to the case in which only YORP is included. However, additional work is needed to explain the significant excess of slow rotators that our model produces, even after an artificial bias is imposed to our model results. There are a number of possible sources for this discrepancy. We may under (or over) estimate the effects on YORP cycles of the topographical changes caused by the cratering events. To have additional clues on this aspect more modeling is needed on the relative change of the YORP coefficient $C_{Y}$ after an impact altering the superficial features of the body. Also, the obliquity evolution is not fully modeled since we neglect the effects of YORP on it. Finally, as discussed in the text, the size distribution of asteroids at the small size end influences the lifetime of asteroids and alters the slow rotator tail. Some aspects of our model can be improved by further independent studies on YORP and the evolution of bodies beyond the critical limit. An analytic theory of how YORP affects the obliquity evolution is needed since at present the obliquity of our sample bodies changes only because of collisions. This may not significantly alter the outcome of our modeling since the YORP obliquity change may be either absorbed by collision variations or by the overall statistics involving many bodies with changing YORP 
coefficients. We also need to outline an algorithm able to statistically select an outcome between binary formation and mass shedding anytime a body in our sample is accelerated to breakup. This is work in progress following the theory presented in Jacobson and Scheeres (2011). Once implemented, this algorithm will allow us to derive the fraction of asteroids which will become objects like P/2010 A2, developing a dusty tail as a consequence of mass shedding, compared to the fraction of binary asteroids and predict the relative number of events per year.

\section{Acknowledgments}

We thank David Vokrouhlický and Alan Harris for productive comments and suggestions while acting as referees of the paper. DJS acknowledges support from NASA's Office of Space Science Planetary Geology and Geophysics Program and the Outer Planets Research Program.

\section{References}

Bottke, W. F., Nolan, M. C., Greenberg, R., Kolvoord, R. A., 1994. Velocity distributions among colliding asteroids. Icarus 107, 255-268.

Bottke, W.F., Vokrouhlický, D., Rubincam, D.P., Brož, M., The effect of Yarkovsky thermal forces on the dynamical evolution of asteroids and 
meteoroids. In: W.F. Bottke Jr., A. Cellino, P. Paolicchi and R.P. Binzel, Editors, Asteroids III, Univ. of Arizona Press, Tucson (2002), p. 405.

Bottke, W. F., Durda, D. D., Nesvorný, D., Jedicke, R., Morbidelli, A., Vokrouhlický, D., Levison, A., 2005. Linking the collisional history of the main asteroid belt to its dynamical excitation and depletion. Icarus 179, 63-94.

Čapek, D., Vokrouhlický, D., 2004. The YORP effect with nite thermal conductivity. Icarus 172,526536 .

Cicaló, S., and Scheeres, D., 2010. Averaged rotational dynamics of an asteroid in tumbling rotation under the YORP torque. Cel. Mech. and Dyn. Astron. 106, 301-337.

Dohnanyi, J.W., 1969, Collisional models of asteroids and their debris, J. Geophys Res. 74, 25312554.

Donnison, J. R., and Wiper, M.P., 1999. Bayesian statistical analysis of asteroid rotation rates. MNRAS 302, 75-80.

Efroimsky, M. and Lazarian, A., 2000. Inelastic dissipation in wobbling asteroids and comets. MNRAS 311, 269-278.

Farinella, P., Davis, D. R., 1992. Collision rates and impact velocities in the Main Asteroid Belt. Icarus 97, 111-123.

Farinella, P., Davis, D. R., Paolicchi, P., Cellino, A., Zappala, V., 1992. 
Asteroid collisional evolution - an integrated model for the evolution of asteroid rotation rates. A\&A 253, 604-614.

Fulchignoni, M., Barucci M.A., Di Martino M., Dotto, E. 1995. On the evolution of asteroid spin. Astron. Astrophys. 299, 929-932.

Gladman, B.J., Davis, D.R., Neese, C., Jedicke, R., Williams, G., Kavelaars, J.J., Petit, J.-M., Scholl, H., Holman, M., Warrington, B., Esquerdo, G., Tricarico, P., 2009, On the asteroid belt's orbital and size distribution, Icarus 202, 104-118.

Ivezic, Z. et al., 2001. Solar System Objects Observed in the Sloan Digital Sky Survey Commissioning Data. AJ 122, 2749-2784.

Jacobson, S.A. and D.J. Scheeres. 2011. Dynamics of Rotationally Fissioned Asteroids: Source of Observed Small Asteroid Systems, submitted to Icarus.

Jewitt, D., Weaver, H., Agarwal, J., Mutchler, M., Drahus, M., 2010. A recent disruption of the main-belt asteroid P/2010A2 Nature 467, 817-819.

Kaasalainen, M., Ďurech, J., Warner, B. D., Krugly, Y. N., Gaftonyuk, N. M., 2007. Acceleration of the rotation of asteroid 1862 Apollo by radiation torques. Nature 446, 420-422.

Nesvorný D., and Vokrouhlický D., 2008. Analytic theory for the YarkovskyO'Keefe-Radzievski-Paddack effect on obliquity. AJ 136, 291-299. 
O’Brien, D. P., Greenberg, Richard, 2003. Steady-state size distributions for collisional populations: analytical solution with size-dependent strength. Icarus 164, 334-345.

Pravec, P., Harris, A. W., Vokrouhlický D., Warner, B. D., and 27 coauthors, 2008. Spin rate distribution of small asteroids. Icarus 197, 497-504.

Richardson, D.C., Quinn, T., Stadel, J. and G. Lake, 2000. Direct largescale N-body simulations of planetesimal dynamics, Icarus 143, 4559.

Richardson, D.C., Elankumaran, P., and R.E. Sanderson, 2005. Numerical experiments with rubble piles: Equilibrium shapes and spins, Icarus 173, 349361.

Rossi, A.; Marzari, F.; Scheeres, D. J., 2009. Computing the effects of YORP on the spin rate distribution of the NEO population. Icarus 202, 95-103.

Rubincam, D.P., 2000. Radiative spin-up and spin-down of small asteroids. Icarus 148, 211.

P. Sánchez and D.J. Scheeres. 2011. "Simulating Asteroid Rubble-Piles with a Self-Gravitating Soft-Sphere DEM Model," Astrophysical Journal, 727: 120 .

Scheeres, D. J., Marzari, F., Rossi, A., 2004. Evolution of NEO rotation rates due to close encounters with Earth and Venus. Icarus 170, 312-323. 
Scheeres, D.J., Fahnestock, E.G., Ostro, S.J., Margot, J.-L. Benner, L.A.M., Broschart, S.B., Bellerose, J., Giorgini, J.D., Nolan, M.C., Magri, M., Pravec, P., Scheirich, P., Rose, R., Jurgens, R.F., Suzuki, S., DeJong, E.M., 2006, Dynamical configuration of binary near-Earth Asteroid (66391) 1999 KW4, Science 314, 12801283.

Scheeres, D. J., 2007. The dynamical evolution of uniformly rotating asteroids subject to YORP. Icarus 188, 430-450.

Snodgrass, C. et al. A collision in 2009 as the origin of the debris trail of asteroid P/2010A2. Nature 467, 814-816.

Statler, T. S., 2009. Extreme sensitivity of the YORP effect to smallscale topography. Icarus 202, 502-513.

Taylor, P. A., Margot, J., Vokrouhlický, D., Scheeres, D. J., Pravec, P., Lowry, S. C., Fitzsimmons, A., Nolan, M. C., Ostro, S. J., Benner, L. A. M., Giorgini, J. D., Magri, C., 2007. Spin Rate of Asteroid (54509) 2000 PH5 Increasing Due to the YORP Effect. Science 316, 274-275.

Vedder, J. D., 1996. Asteroid Collisions: Estimating Their Probabilities and Velocity Distributions. Icarus 123, 436-449.

Vedder, J. D., 1998. Main Belt Asteroid Collision Probabilities and Impact Velocities. Icarus 131, 283-290.

Vokrouhlický, D., Čapek, D., 2002. YORP-induced long-term evolution 
of the spin state of small asteroids and meteoroids. Rubincams approximation. Icarus 159, 449467.

Vokrouhlický, D., Breiter, S., Nesvorný, D., Bottke, W. F., 2007. Generalized YORP evolution: Onset of tumbling and new asymptotic states. Icarus 191, 636-650.

Walsh, K. and Richardson, D.C., Steady-state population of the NEA binaries and YORP spinup models, 2006, Bull. Am. Astron. Soc. 38 Abstract 53.08. 

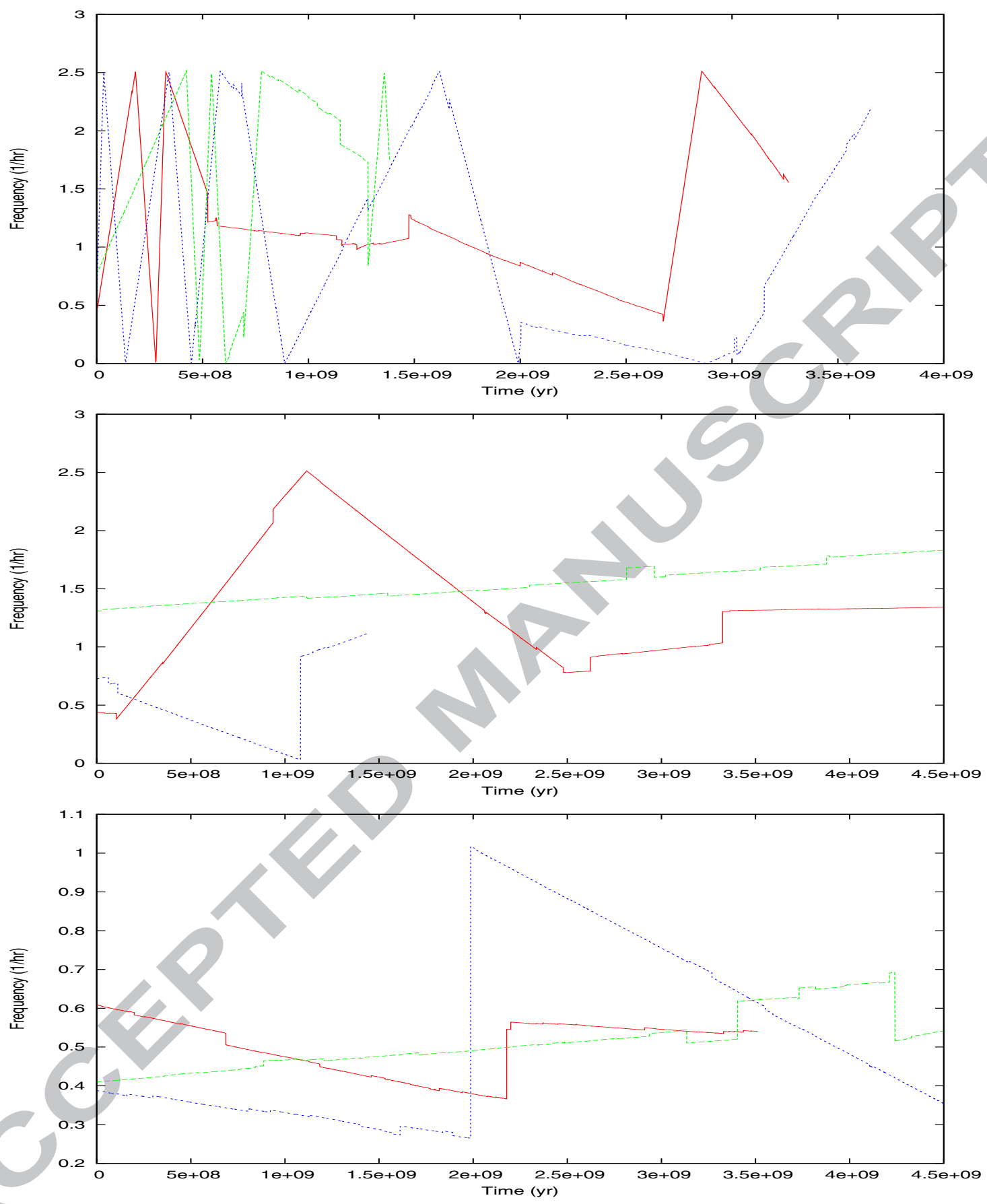

Figure 1: Evolution of the rotation frequency of small asteroids with time. The top plot shows the evolution of 3 individual $1 \mathrm{~km}$ size object, the middle plot that of $5 \mathrm{~km}$ size bodies and the bottom plot for $10 \mathrm{~km}$ size bodies. Different color lines within the same plot indicate objects with different initial parameters. The selected cases are not a statistical representative sample in terms of collisional lifetime. 


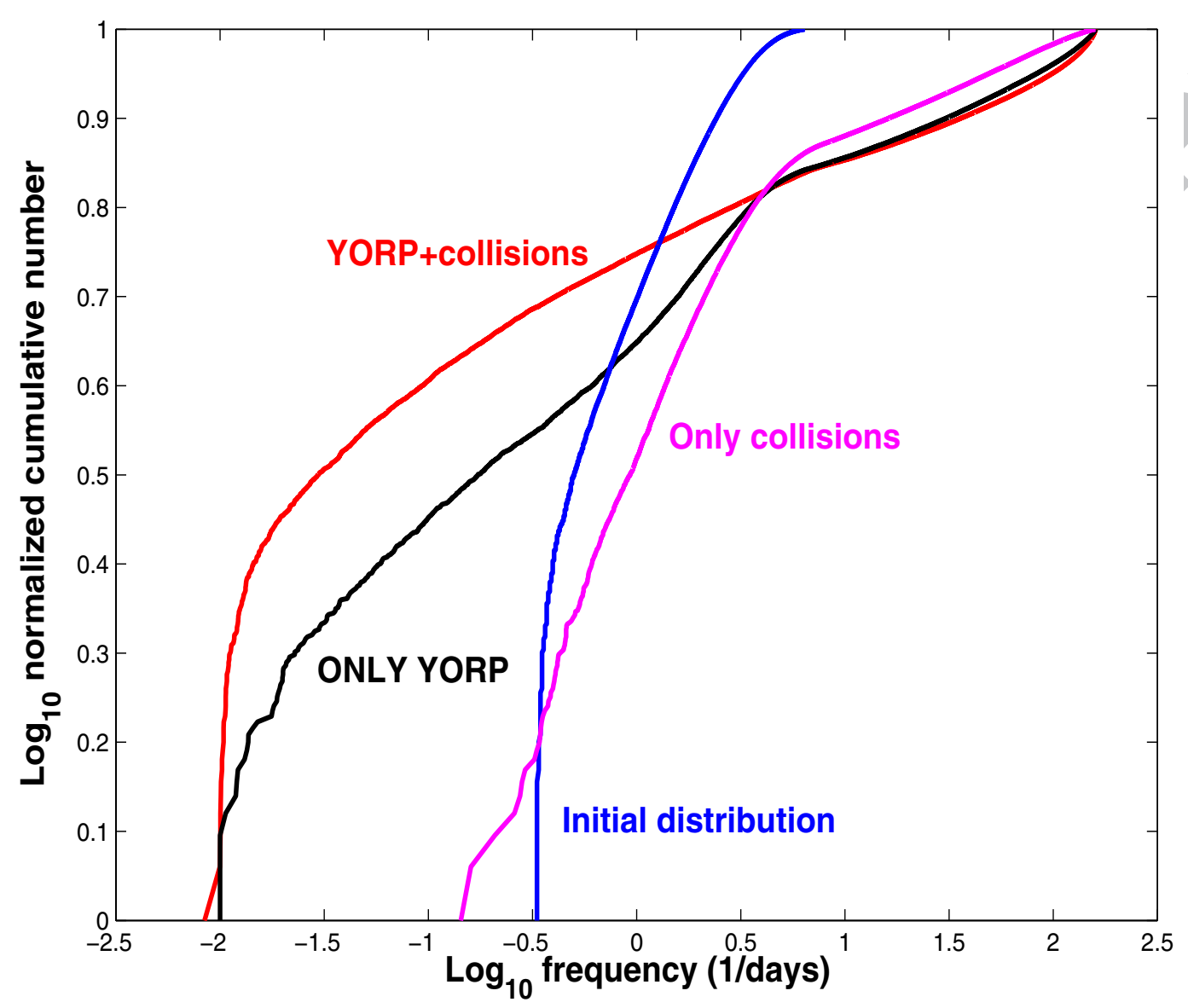

Figure 2: Cumulative rotation rate distribution of small Main Belt asteroids after $4.5 \times 10^{9}$ years of evolution. To better match observations, the model population has been artificially biased in size as in Rossi et al. (2009). The diamter range spanned by our model has been divided in logarithmic size bins and in each of this bin a number of bodies equal to the observed ones have been randomly selected within our numerical sample. 


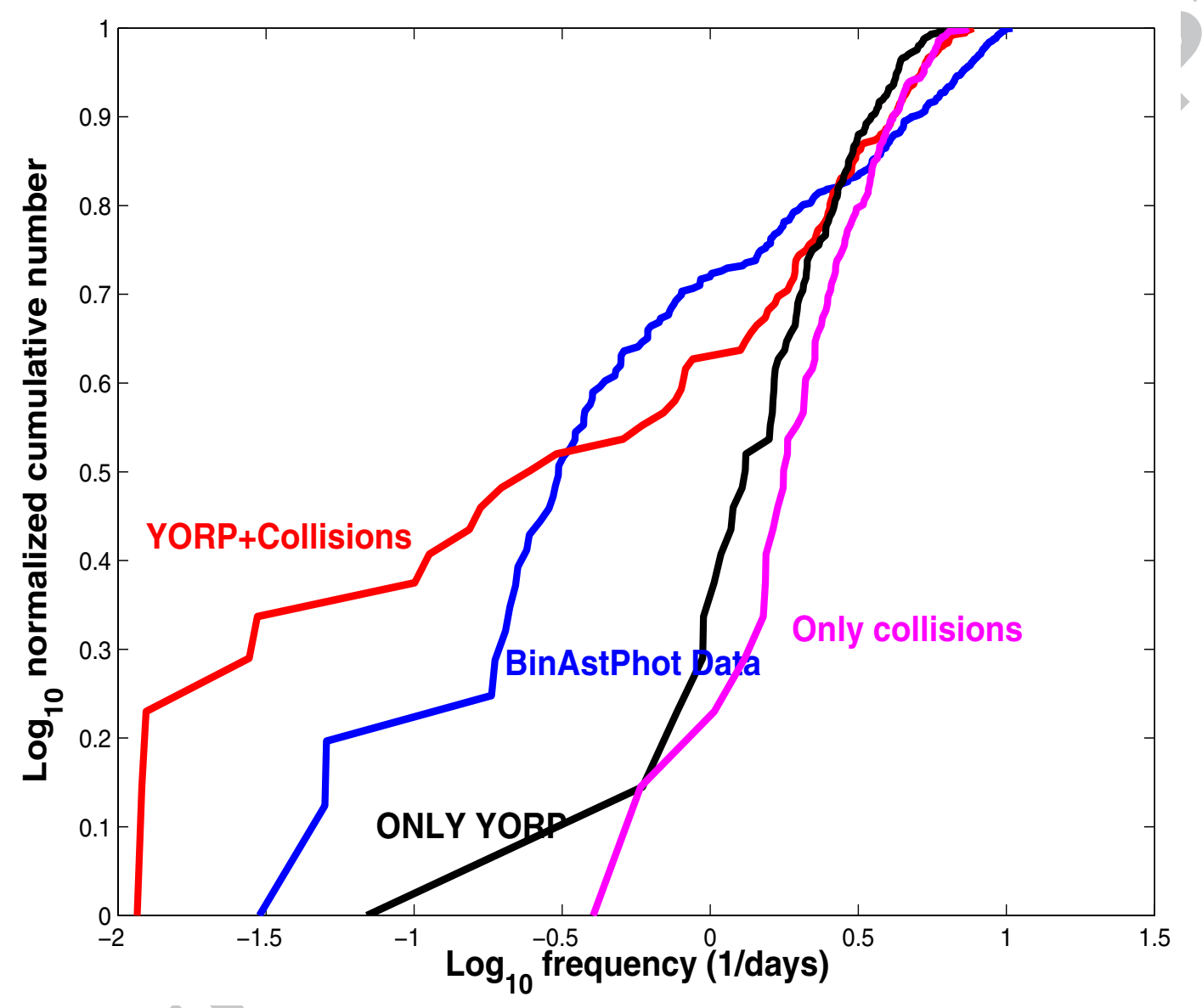

Figure 3: Same as in Fig. 2 but an artificial bias is applied to the model results to better reproduce observations. 


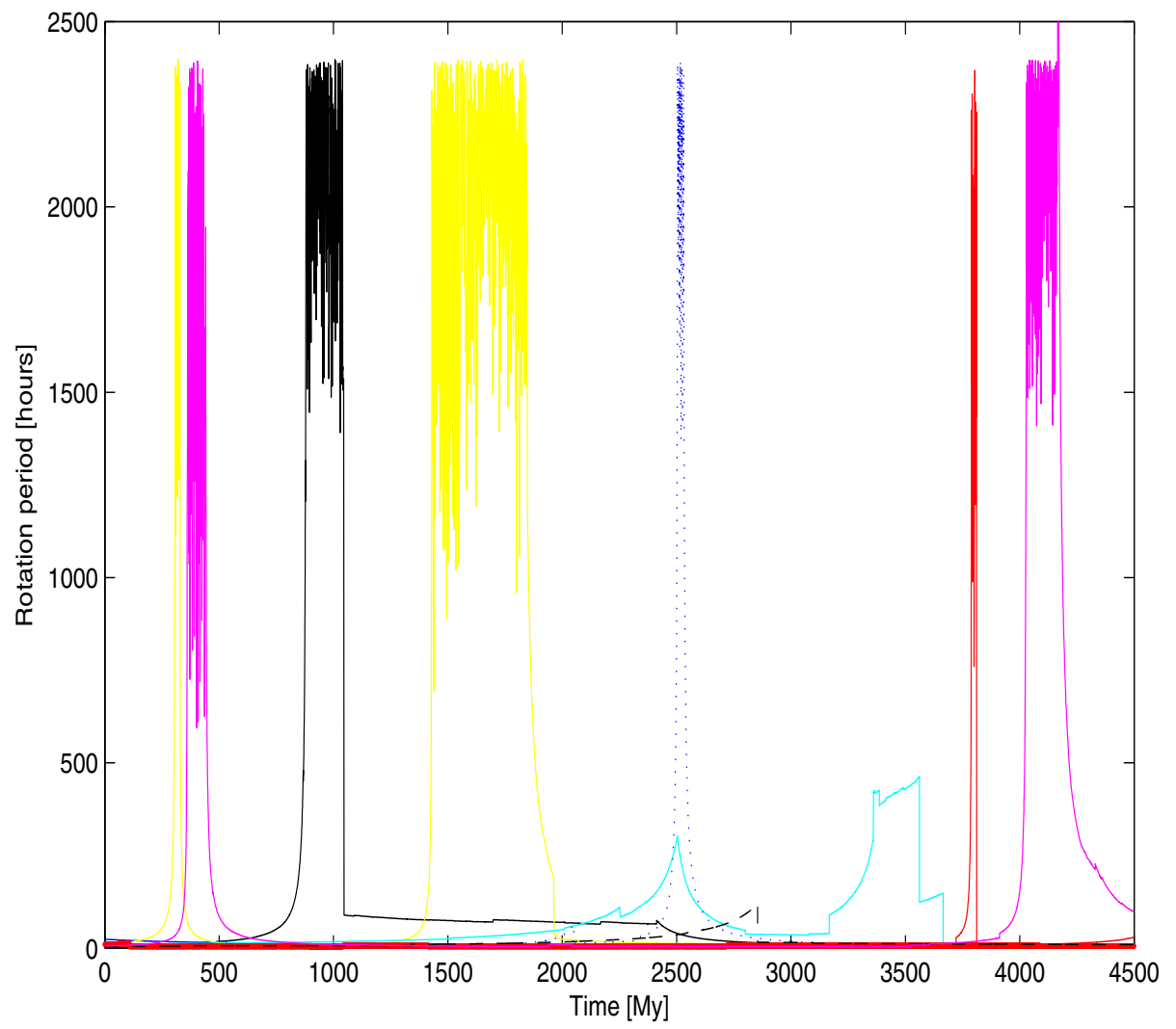

Figure 4: Time evolution of the spin period for a few large objects within our sample, under the effect of YORP and collisions. 


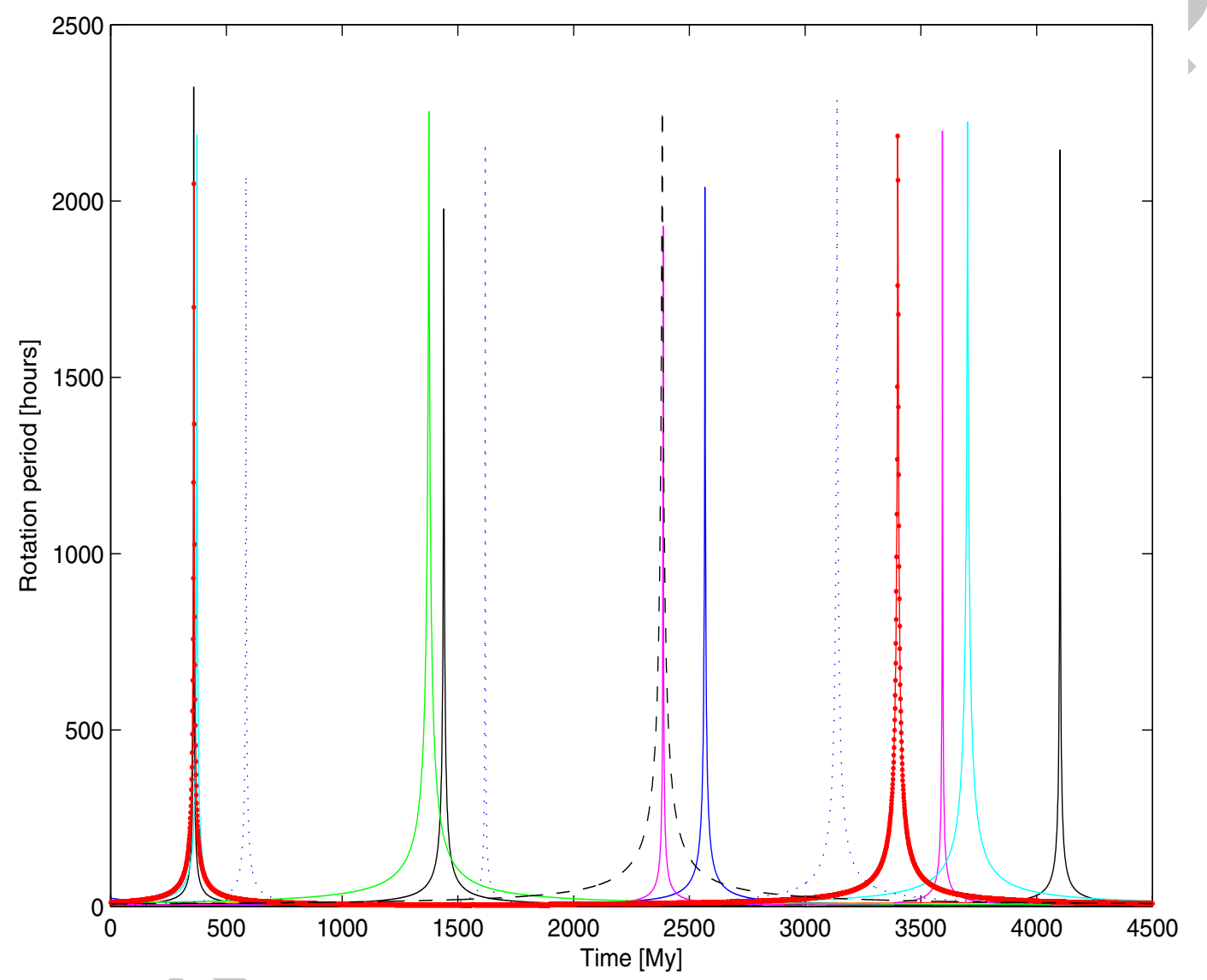

Figure 5: Time evolution of the spin period for the same objects shown in Fig. 4, under the effect only of YORP. 


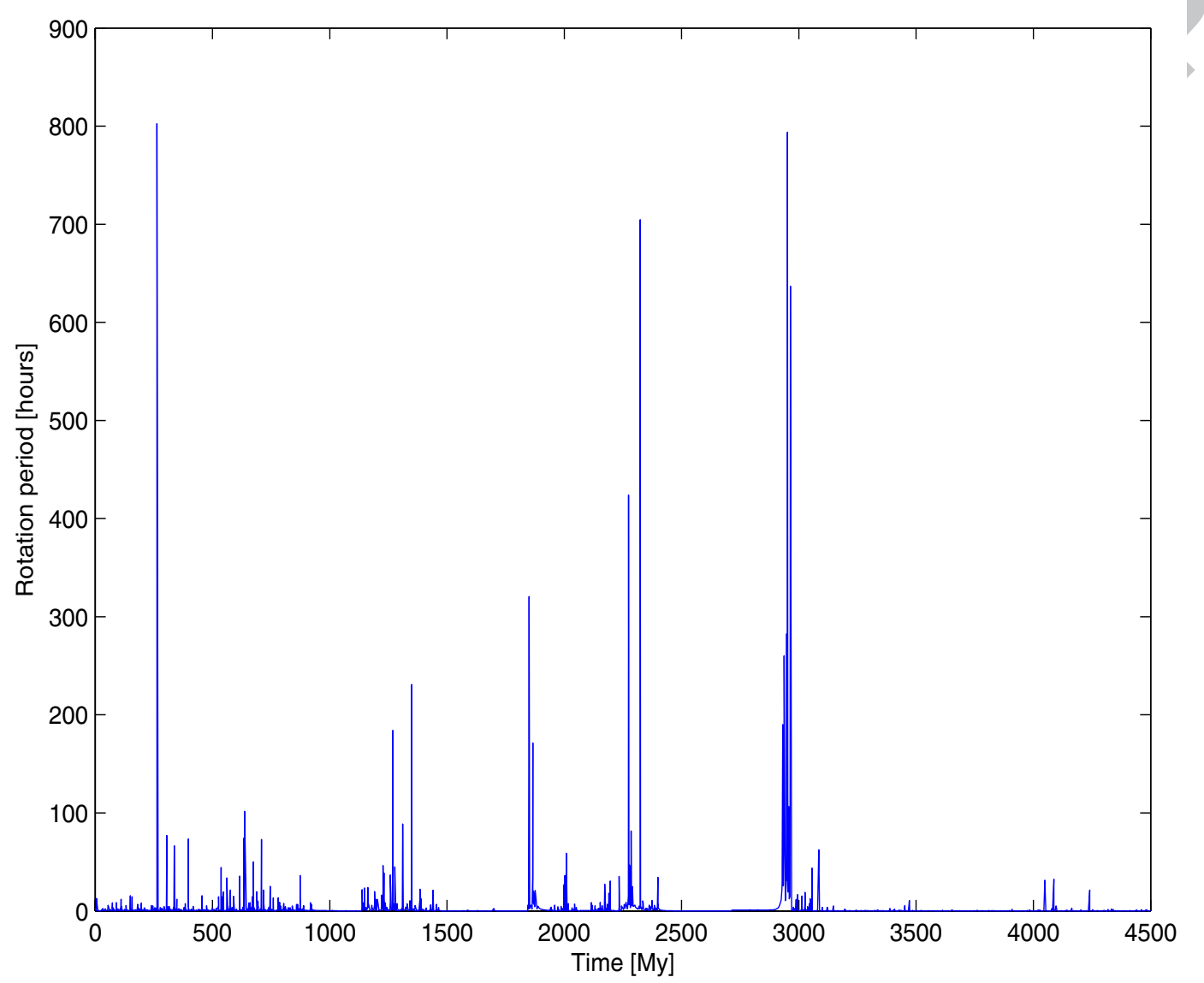

Figure 6: Time evolution of the spin period for a small object within our sample, under the effect of YORP and collisions. 


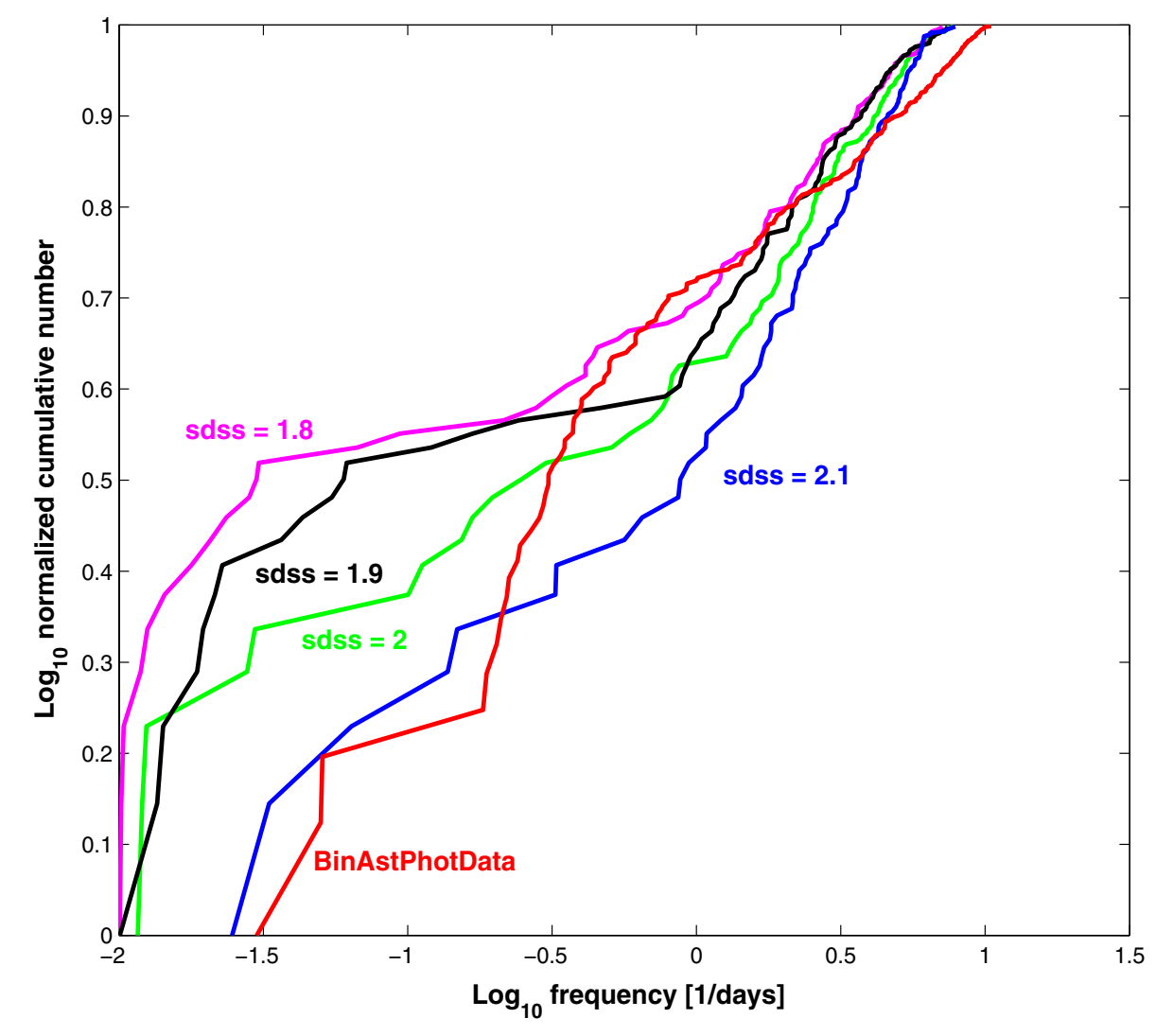

Figure 7: Same as in Fig. 3 but different slopes for the size distribution of small asteroids are used. 


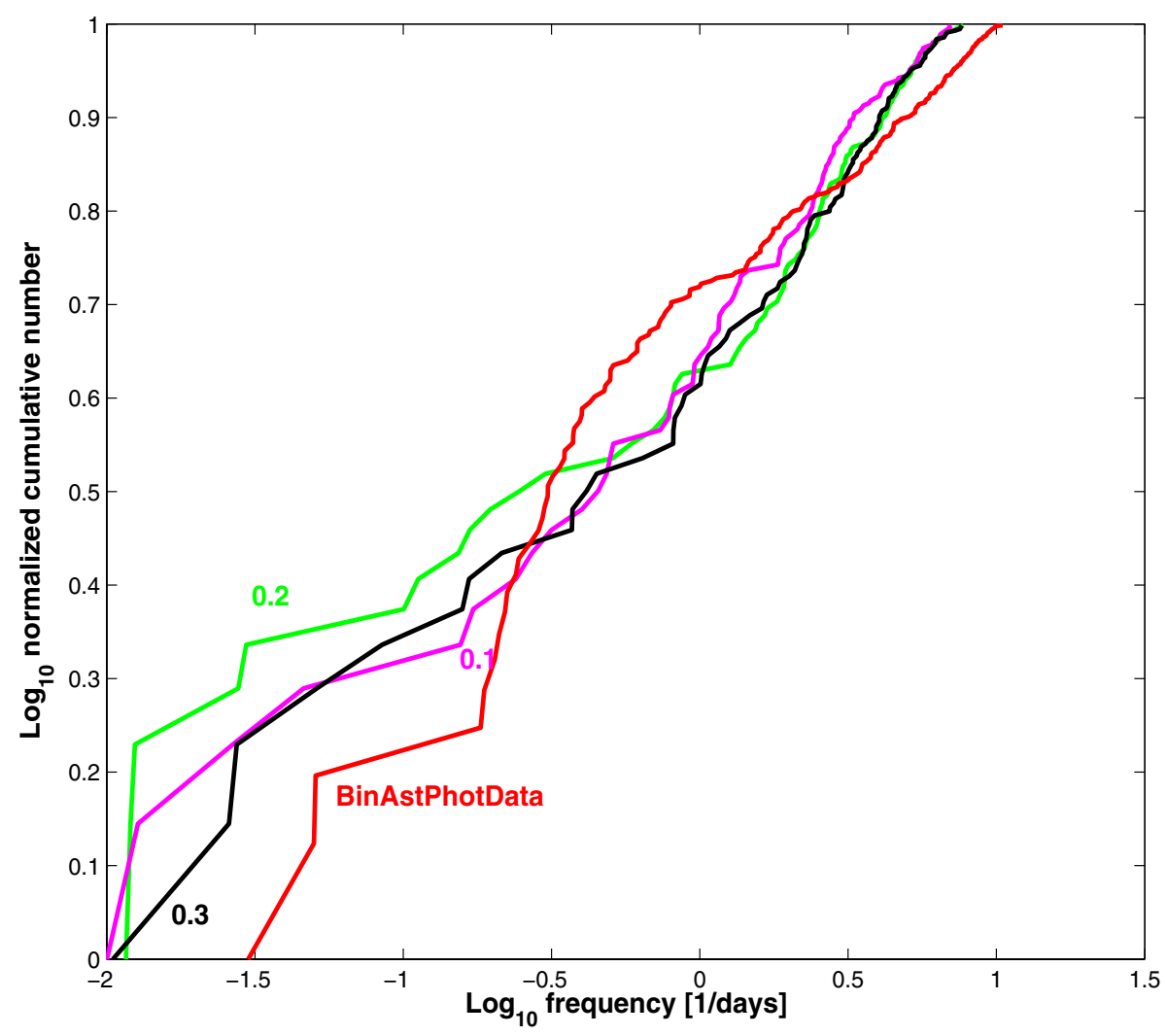

Figure 8: Same as in Fig. 3 but different values of the parameter $\delta=$ $0.1,0.2,0.3$. 


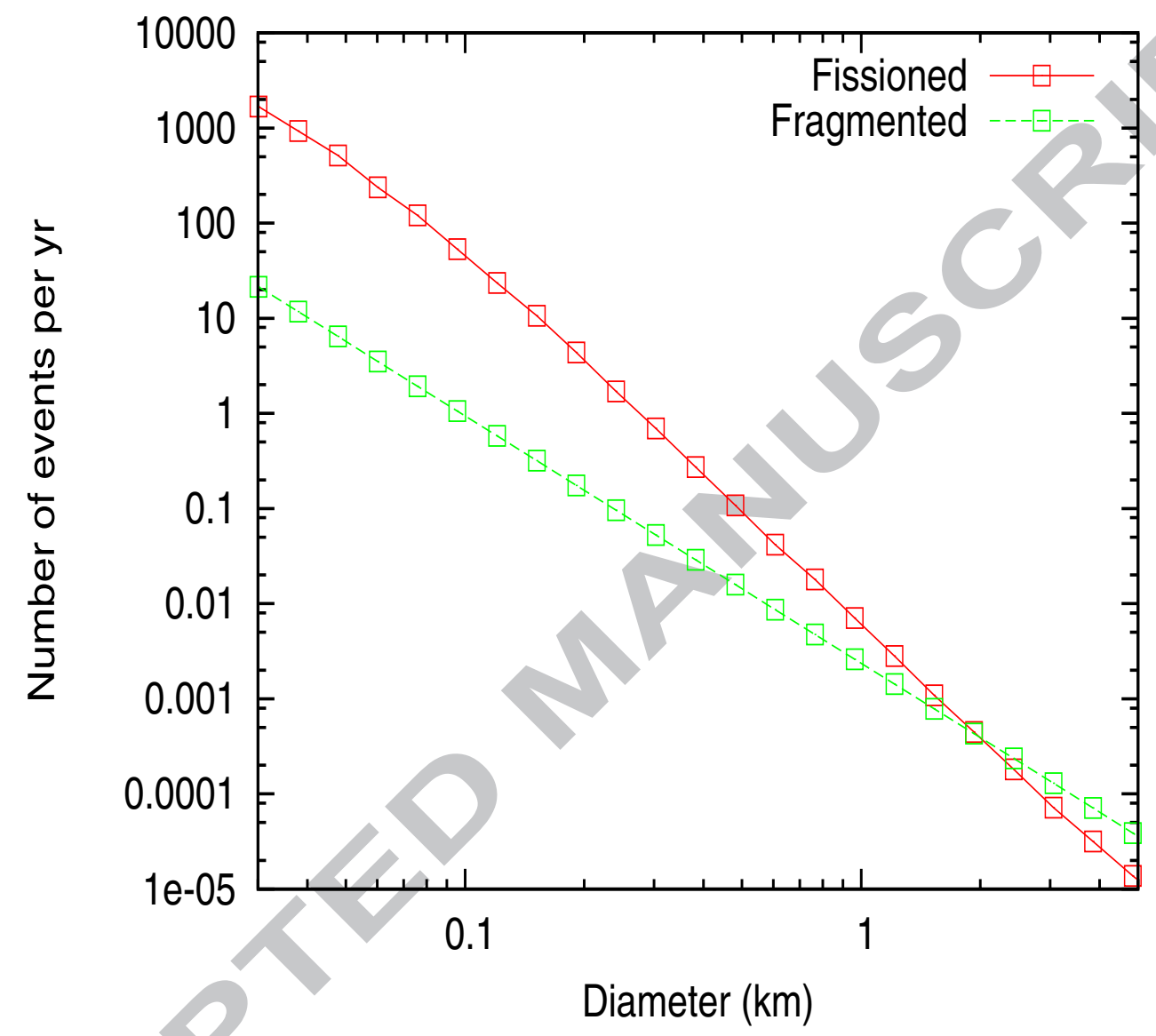

Figure 9: Number per year of expected small asteroids which will be spun up to fission in the asteroid belt as a function of their diameter. For comparison, the number of breakup events is shown. 
In this study we model the evolution of the rotation rate of small Main Belt asteroids under the effects of YORP and collisions.

We find that cratering collisions coupled to YORP give non-linear effects which depend on the size of the bodies and are particularly relevant at slow rotation rates. These effects lead to the observed excess of slow rotators among small asteroids.

Our model also shows that the rotational fission of small asteroids is a very frequent event and might explain objects like P/2010 A2 and its associated tail of millimeter-sized dust particles. 